\title{
Simulation Data Analysis Using Fuzzy Graphs
}

\author{
Klaus-Peter Huber and Michael R. Berthold \\ Institute for Computer Design and Fault Tolerance (Prof. D. Schmid) \\ University of Karlsruhe - P.O. Box 6980 - 76128 Karlsruhe - Germany \\ eMail: [kphuber,berthold]@Informatik.Uni-Karlsruhe.de
}

\begin{abstract}
Analysis of simulation models has gained considerable interest in the past. However, their complexity still remains a considerable drawback in practical applications. A promising concept is to analyze the data from simulation experiments. Existing approaches are either restricted to simple models or are hard to interpret. We present an efficient algorithm that constructs a fuzzy graph model from simulation data and we show that the resulting system approximates also complex model functions with an adjustable precision. In addition the Fuzzy Graph allows the analyst to directly access easy to interpret if-then-rules. These rules help to understand the original simulation model, which is shown with a real world token bus model.
\end{abstract}

\section{Introduction}

The use of modeling and simulation techniques has gained considerable influence for the development or the optimization of large systems. Unfortunately the complexity of the corresponding simulation model increases with the complexity of the real systems. This leads to several drawbacks: simulation becomes a highly time consuming task which makes it impossible to perform interactive simulations and in addition the analysis of the resulting simulation model is extremely complicated.

A common solution is to build an auxiliary model, the so-called metamodel which is less complicated and therefore easier to interpret. Depending on the task of analysis there may exist different kinds of metamodels for example to analyze the parameter sensitivity [5], or to optimize models [7]. In all cases the generation of a metamodel helps to reduce the complexity of the model that is being analyzed.

Of particular interest are approaches that build the metamodel solely through observations of the original model, i.e. through analysing data from simulation experiments (Figure 1). One way is to analyze the data with statistical methods to find some unknown dependencies but in high-dimensional feature space the mathematical solution is not trivial and the interpretation is not straightforward. 


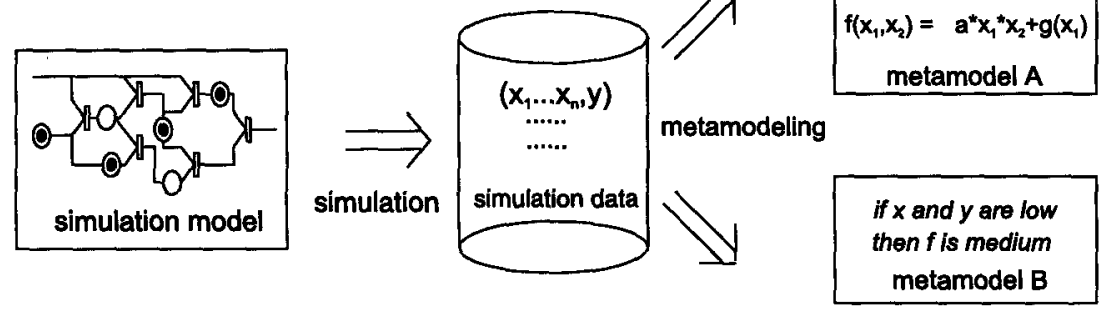

Fig. 1. Data based Metamodeling Process

Other approaches use methods from the machine learning area and find a set of rules or a decision tree.

In this paper we present an approach that analyses data resulting from simulation experiments and constructs a so-called Fuzzy Graph. This allows an easy interpretation of the input-output behavior since the metamodel can be represented with fuzzy rules. Analysis of the rules helps to uncover the underlying dependencies between factors and one output parameter and the rule base can also be used as a fast simulator.

\section{Building a Metamodel from Data}

Here, we concentrate on models where the behavior can be described by a function

$$
f_{\text {model }}(\mathbf{x})=f_{\text {model }}\left(x_{1}, \cdots, x_{n}\right)=y
$$

where $n$ factors $x_{1}, \cdots, x_{n}$ are considered and $y$ is the output parameter of interest. In practice this function can not be extracted explicitly from the model description and is therefore unknown. To build a suitable metamodel means to perform $m$ experiments with the model resulting in $m$ data points $\left(x_{1}^{i}, \cdots, x_{n}^{i}, y^{i}\right)$ $(i \in[1, m])$ and to build a metamodel representing a function $f_{\text {meta }}\left(x_{1}, \cdots, x_{n}\right)=$ $\tilde{y}$ with $\tilde{y}$ being the approximated value of $y$. If the data is generated by a stochastic simulation process, $y$ is a stochastic variable. There may exist several different values of $y$ for the same input parameters, e.g. by simulation with different random number streams. This should be taken into account when building a metamodel. To validate the quality of the metamodel the difference between the observed $y$ values and the approximated values $\tilde{y}$ can be evaluated.

Metamodeling approaches can be categorized into statistical approaches that use the data to adapt a special kind of function with statistical means and Machine Learning Algorithms that use the data to generate a rule set.

\subsection{Statistical Approaches for Metamodeling}

Ad hoc methods use handfitted curves or graphical approaches, other methods use linear regression models with a least square approach to define the parameters [6]. Regression functions are very popular since the resulting models are 
easy to handle and interpret, and statistical methods like t-tests and F-tests can be used to validate the quality of the model [2]. To deal with nonlinear behavior metamodeling regression functions are often defined as:

$$
f_{m e t a}(\mathbf{x})=\beta_{0}+\sum_{j=1}^{n} \beta_{j} \cdot x_{j}+\sum_{i, l} \beta_{i, l} \cdot x_{i} \cdot x_{l}+\epsilon
$$

with $\epsilon$ representing an error term and $\beta_{i, l}$ representing some user-defined in pair dependencies. To find a "best fitting" metamodel the regression parameters $\beta_{0}, \beta_{j}$ and $\beta_{i, l}$ can be obtained by minimizing the mean square error in respect to the example data. The resulting function can be used for approximation or for analysis, e.g. to obtain some information about the sensitivity of each factor. But in practice often the behavior of a simulation model can not be described with such simple first-order models (see for example [2]).

\subsection{Machine Learning Approaches for Metamodeling}

To avoid the need for background knowledge nowadays several methodologies have been proposed that make use of rule learning algorithms from the machine learning area. In [7] preprocessed (i.e. cleaned and digitized) data from the simulation model was used to directly extract decision rules and in [5] a similar approach was used for sensitivity analysis. Both concepts are based on methods that build a classifier so they are restricted to applications where the output parameter is not continuous.

Another kind of learning techniques that are especially well suited to deal with "noisy" or "stochastic" data originate from the Soft Computing or Fuzzy Systems area [11]. They offer an easy way to model soft data points, for example values with a corresponding confidence interval. Unfortunately, most known methods that build fuzzy systems from data have severe limitations in this context. Some require an a priori defined set of rules that is just fine tuned during training $[9,10]$, others construct the ruleset but the result depends heavily on the order of training examples [8]. A more sophisticated algorithm [3] tries to divide individual attributes step by step using an increasing number of membership functions but tends to split the feature space into too many tiles. For the purpose of data analysis and metamodeling it is of much more interest, however, to find only few rules that cover a large portion of the feature space.

In the following a new approach is proposed that allows the usage of Fuzzy Graphs [11] to represent the discovered knowledge.

\section{Data Driven Construction of Fuzzy Graphs}

The definition and interpretation of a Fuzzy Graph is manifold. Here, we use it to represent a function with means of Fuzzy Logic instead of mathematical equations, analogous to [11]. The domains of the input parameters and the domain of the output parameter are described with so-called linguistic variables, represented through a set of individual membership functions. The mapping from 


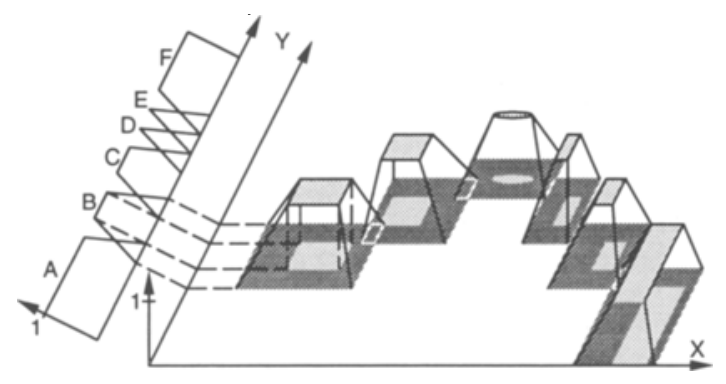

Fig. 2. A One-dimensional Fuzzy Graph

input to output is defined by a collection of Fuzzy Points. Well-known techniques like the center-of-gravity calculation allow to approximate real valued functions based on this Fuzzy Graph. Here, a Fuzzy Graph consists of a collection of Fuzzy Points that can be represented as if-then-rules. Other ways to represent Fuzzy Graphs are described in [11]. The main advantage of the Fuzzy Graph concept is the very compact and easy to understand representation of a function.

The algorithm presented in this section automatically constructs a Fuzzy Graph based on a set of data examples. Fuzzy Graphs built by our approach use a fixed soft granularization on the output variable which means that the user defines the membership functions of $y$ a-priori. This is helpful to focus automatic generation of the Fuzzy Graph on specific regions of the output value and to weaken constraints (and therefore the evolving number of rules) on regions with a low focus of attention. Figure 2 shows an example where the output variable $y$ was already partitioned into 6 soft regions with user defined membership functions. The partitioning of the input variables is determined from the data examples.

Each Fuzzy Point corresponds to one region of the output parameter and is described by its core-region and a larger support-region. The core represents the smallest area where data examples of the corresponding output-region were found and therefore is given a membership value of 1 . The larger support-area contains no examples of other regions, and towards its boundaries the membership value declines linearly to 0 . This leads to the trapezoidal membership functions illustrated in Figure 3.

Each such Fuzzy Point represents exactly one if-then-rule that can be described as:

$\begin{array}{ll}\text { IF } \quad x_{1} & \in\left[b_{1}, c_{1}\right] \subset\left(a_{1}, d_{1}\right) \\ \text { AND } \cdots & \\ \text { AND } x_{n} & \in\left[b_{n}, c_{n}\right] \subset\left(a_{n}, d_{n}\right) \\ \text { THEN } & y \text { is of } k \\ \text { (weight: } w \text { ) } & \end{array}$

The input parameters are restricted through core $\left[b_{i}, c_{i}\right]$ and support-regions $\left(a_{i}, d_{i}\right)$ with $a_{i}<b_{i} \leq c_{i}<d_{i}$, and each rule corresponds to one region $k$ of 


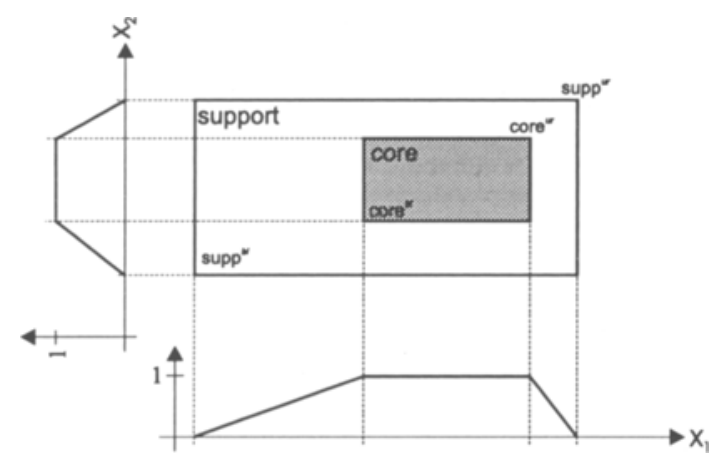

Fig. 3. A Two-dimensional Fuzzy Point

the output parameter. Additionally, the rule weight shows the number of data examples that are covered by the core of the rule and is therefore an indication for the reliability of the rule.

\subsection{Automatic Construction of the Fuzzy Graph}

Construction of the Fuzzy Graph is done using a set of data examples. Each of these examples consists of values for the input parameters with a corresponding output. The output value (or target) is given as a soft value, using an individual membership function $\mu_{T}$. For practical applications these soft targets can originate from stochastic simulation experiments. For sharp targets a singleton can be used as the corresponding output membership function. From this soft target membership function $\mu_{T}$ the membership values $\mu_{k}$ for each of the pre-defined output-regions $k(1 \leq k \leq c)$ are computed using a fuzzy and-operator (min) between the target $\mu_{T}$ and the $k$-th region membership set $\mu_{k}$. This leads to example data consisting of an input vector $\mathbf{x}=\left(x_{1}, \cdots, x_{n}\right)$ with the corresponding $\boldsymbol{\mu}=\left(\mu_{1}, \cdots, \mu_{c}\right)\left(0 \leq \mu_{i} \leq 1\right)$ where $c$ denotes the number of predefined soft regions $y$ is divided into.

The method presented in this paper makes sure that each data example is covered by a rule (Fuzzy Point) of the region with the highest membership value and that rules of regions with membership values $=0$ do not cover the example. This is useful to tolerate moderately noisy patterns or small oscillations along region boundaries. The basic algorithm for one epoch, i.e. one presentation of all data examples, is presented in figure 4 . The algorithm is based on three steps that introduce new rules when necessary and adjust the core- and support-regions of existing rules:

- covered: if a new data example lies inside the support-region (defined through supp ${ }^{b l}$ and supp ${ }^{u r}$ ) of an already existing rule belonging to the region with the maximum $\mu_{k}$, the core-region (core ${ }^{b l / u r}$ ) of this rule is extended to cover the new example, which is, even in high-dimensional space, an easy task. 


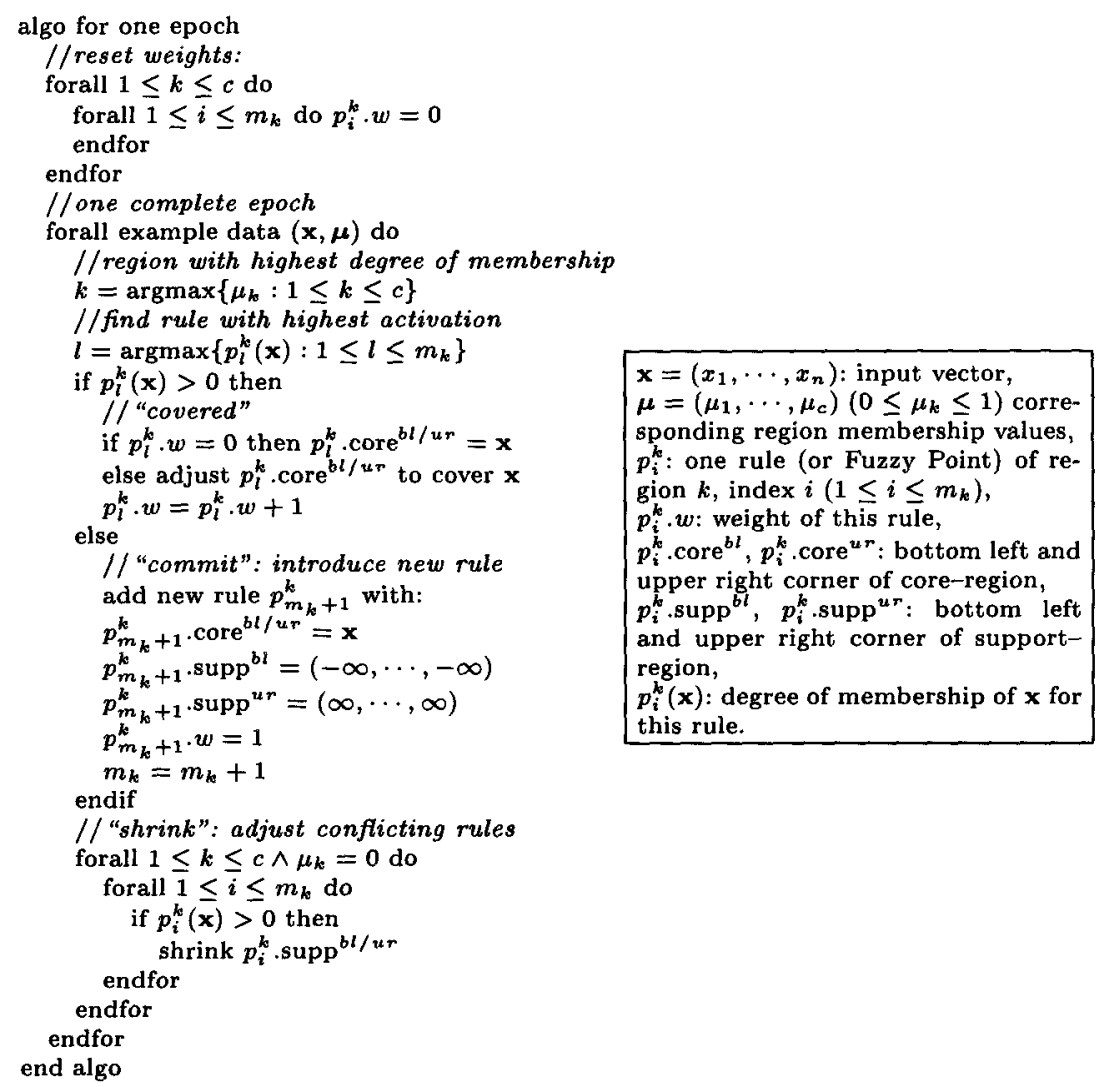

Fig. 4. The Fuzzy Graph Algorithm

- commit: if a new example is not covered, a new rule with the region corresponding to the maximum $\mu_{k}$ will be introduced. The core-region is defined by this new example and the support-region is set infinite.

- shrink: if a new example is incorrectly covered by an already existing rule of a conflicting region $\left(\mu_{k}=0\right)$, this rule's support-area will be reduced (e.g. shrunk) so that the conflict is solved. Here, a heuristic is used that tries to maximize the volume of the remaining support area. See [4] for details.

The whole generation process usually takes only about 4-5 epochs until the structure of the Fuzzy Graph automatically stops to change. Two conditions will hold for all example data $(\mathbf{x}, \boldsymbol{\mu})$ after generation of the Fuzzy Graph: There is at least one rule of region $k$ with the highest membership value $\mu_{k}$ which has $\mathbf{x}$ inside its core. And for all regions $k$ with $\mu_{k}=0, \mathbf{x}$ lies outside of their support area. This leads to a rule base where each example data is covered by an appropriate rule and not covered by those of conflicting regions. This resulting Fuzzy Graph can now be used for approximation. 


\subsection{Fuzzy Graph Function Approximation}

To use the constructed Fuzzy Graph for function approximation for a given input parameter $\mathbf{x}$ the membership degree for each region of the output variable is typically computed using the maximum-operator:

$$
\mu_{k}(\mathbf{x})=\max _{i}\left\{p_{i}^{k}(\mathbf{x}): 1 \leq i \leq m_{k}\right\} .
$$

These values are then used to determine the resulting output membership function. If a crisp value is required, a defuzzification technique can be used, for example Center of Gravity. The combination of the respective parts of the membership functions $\mu_{k}$ leads to the soft value defined through $\mu_{y}$ :

$$
\mu_{y}(\mathbf{x})=\max _{k}\left\{\mu_{k}(\mathbf{x})\right\} .
$$

Computing the center of this function leads to the crisp approximation $y$.

Different experiments with a variety of data sets show that the resulting Fuzzy Graph approximates the original function well, with a specific degree of accuracy (see [1] for experiments with different data sets). In regions containing "noise", the Fuzzy Graph ignores the oscillations and tends to produce plateaus. The degree of noise tolerance depends mainly on the width of the membership functions for the output parameter. Thus the amount of smoothing can be controlled by the output fuzzification. Using more and finer membership functions results in higher precision but forcing a system to follow the data points very closely, will result in a large number of rules that model the noise.

In the following an experiment with a complex model shows the usefulness of the presented approach for the analysis of simulation data.

\section{Example of a Token Bus}

To demonstrate how the presented approach can be used to find rules in data resulting from experiments, a real world simulation model was chosen. The used Token Bus system belongs to the class of field bus systems, i.e. a special type of communications systems, designed to connect machines and computers in a manufacturing environment. Important requirements in this area are "real time" facility, high flexibility, and low costs. In this section the analysis will mainly focus on the real time facility of the model, that is its capability to respond to each request within a limited time. To guarantee this property for the given simulation model a metamodel was built using the presented method and its behavior depending on different parameter settings was explored.

The modeled token bus system corresponds to the seven level architecture of the ISO/OSI communication standard. Figure 5 shows the structure of the system. Many details like different message priorities and the token handling had to be taken into account when modeling the system with a queuing network model. The model was then implemented with a commercially available simulation environment. To illustrate the complexity of the underlying queuing 


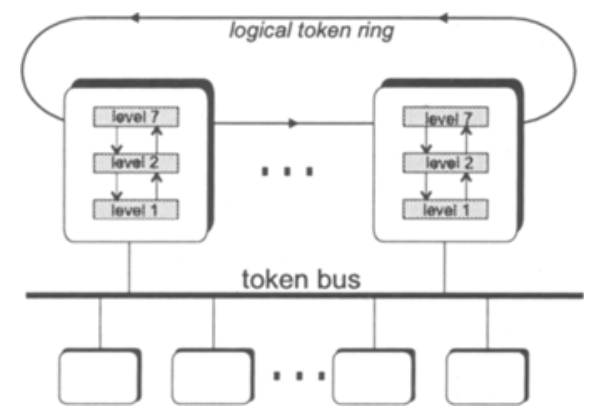

Fig. 5. The Model

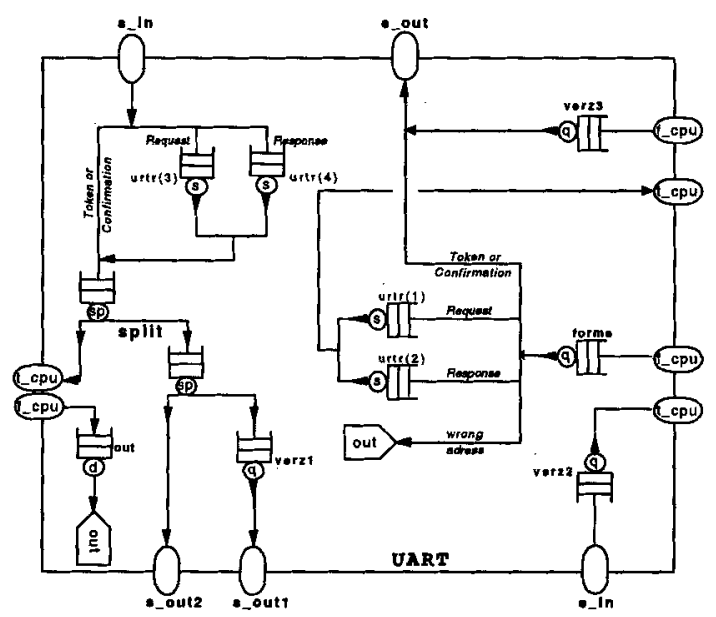

Fig. 6. The Internal Structure of one Module

network the internal structure of one module with its interfaces is illustrated on Figure 6. Since each station is modeled by four different modules the whole model consists of more than two hundred different queues and several hundreds connections. Due to the complexity of the internal structure a conventional analysis of this model is extremely time consuming and complicated.

\subsection{Data Generation and Analysis}

Due to the large number of parameters (20 input and 10 output parameters) of the complete model the example analysis presented in this section will focus on the response time between two master stations in dependence of a selection of parameters of interest. It is desirable that this response time always stays below an upper bound to guarantee that the reaction of the system is always in time. Four input parameters were chosen while the other parameters remained fixed: 
- average time for execution (cpu1): describes the performance of the CPU module of station 1 , i.e., the average time required to execute one command. This value is varied within 0.1 (fast) -3.4 (slow).

- workload rate (workload): describes the average idle time between two requests, this value is varied within 0.02 (low idle time, high workload) -1.0 (low workload).

- maximum target-rotation-time (trt): maximum allowed time to process the token. This parameter controls the time each station has to send messages, values were set within $[0.01,0.4]$

- number of additional stations (stations) represents the background workload on the network. Many additional stations communicating over the network will increase the traffic on the network: $[1,15]$

Since the construction of the metamodel only depends on the example data these examples have to be representative. For this the planning of simulation experiments must be done carefully. In our application a full factorial design is not possible, therefore we used randomized settings for the input parameters. 350 simulation experiments were performed where the input parameter values were varied randomly within the given intervals. The averaged response time $(r t)$ was measured within $(0.088,9.75)$. Each simulation experiment was repeated five times with a different random number stream of the simulation tool. From these five values the minimum, the maximum, and the average were taken and a triangular target membership function was generated (Figure 7). With the Fuzzy

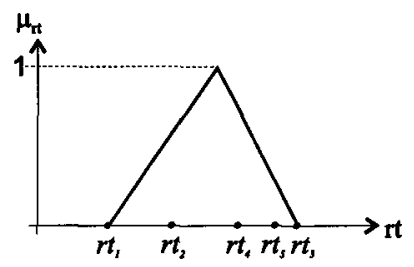

Fig. 7. Generation of a Soft Target

Graph approach these four-dimensional data-vectors with their corresponding target membership function were used for training. Since the main focus of attention were fast responses (i.e. low values of $r t$ ) the membership functions for low values are defined finer than those for bigger values (Figure 8 ). Three series of experiments were performed with two, five and ten membership functions. Fuzzy Graph construction required about 10 seconds on a SUN Sparc10 workstation. No training parameters besides the a priori definition of the output membership functions had to be considered or tuned. While a simulation run takes about 200 seconds the propagation of a new parameter set through the Fuzzy Graph is completed within fractions of a second, resulting in an increase in speed of two orders of magnitude. As expected the metamodel can be used for much faster simulation. 

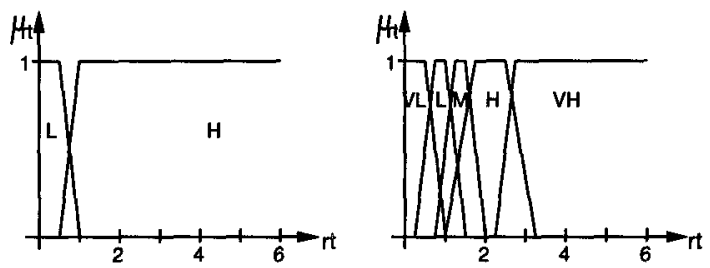

Fig. 8. Two Used Types of Membership Functions

\subsection{Results}

To judge the reliability of the complete rulebase the quality can be analyzed by computing the mean relative error of the approximation of the metamodel. For this analysis an independent dataset that was not used for building the Fuzzy Graph, the so called cross-validation set, was used. The dataset of 350 vectors was split into one tenth for testing and nine tenth for building the graph and using each tenth once for testing ten cross-validation runs were performed. The average error on the corresponding testdata was $4.4 \% \pm 1.0 \%$ (avg. 32 rules) with two, $4.1 \% \pm 1.2 \%$ (avg. 59 rules) with five, and $3.3 \% \pm 0.9 \%$ (avg. 68 rules) with ten membership functions for the output. This approximation quality is sufficient because the primary goal of the presented approach is the extraction of few understandable rules instead of achieving minimal approximation errors.

One of the resulting rule bases from an experiment with two output regions was used for further analysis. In this case the regions are labeled low and high. Since the main focus of analysis were parameter settings which result in a low response time, rules of region $\mathrm{L}=$ low were investigated. From 29 rules 16 belong to this region and according to the rule weight the most important rule was:

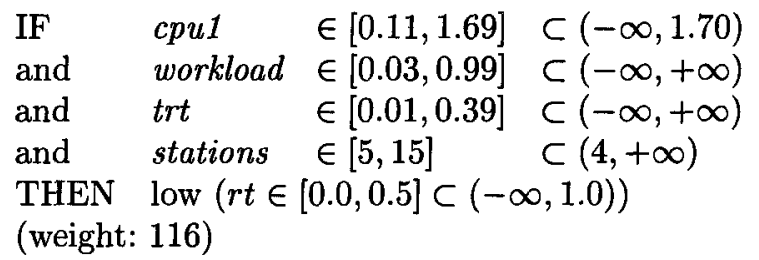

This rule demonstrates how the core always covers a confident subset of the support-region. Here, for two parameters, namely workload rate and targetrotation-time, the core covers the whole range of these parameters. Additionally it is only limited into one direction on the other two parameters, indicated by a support region having finite boundaries. The performing time of CPU1 has to be below 1.70 and the number of additional stations above 4 . This indicates that a certain amount of computation power together with some background stations guarantees fast responses no matter what settings are chosen for workload rate and performing time. In addition the weight of this rule can be used to judge its reliability. The weight indicates the number of examples that are covered by this rule. In the rule shown above 116 examples fall inside its core region. 
This means that about $36 \%$ of all examples are covered by this rule, indicating a high reliability. Rules with low weight on the other hand might be indicators for outliers, irregularities in the dataset or regions of high sensitivity, i.e. regions where small changes of the attributes result in large variations of the output. Another question of interest is the influence of some parameters considering the output. In this example the rule above indicates that the target-rotation-time has no influence on the response time if the CPU is fast (below 1.7) and at least 5 background stations exist because the support of trt is not restricted and the core covers nearly the whole domain of this parameter.

It can also be of interest to find "bad" examples, i.e. regions where the response time is very high. These indicate parameter settings that should be avoided. For example, the rule with the highest weight for response time $=$ high was:

$\begin{array}{llll}\text { IF } & \text { cpu1 } & \in[2.71,3.39] & \subset(2.70,+\infty) \\ \text { and } & \text { workload } & \in[0.09,0.98] & \subset(-\infty,+\infty) \\ \text { and } & \text { trt } & \in[0.14,0.39] & \subset(0.13,+\infty) \\ \text { and } & \text { stations } & \in[1,15] & \subset(-\infty,+\infty) \\ \text { THEN } & \text { high }(r t \in[0.5,1.0] \subset(1.0,+\infty)) \\ \text { (weight: } 38) & & \end{array}$

This rule indicates that if the CPU is very slow and the target-rotation-time is above a certain value the response time is high no matter what workload is considered (represented by background stations and the time between requests). Therefore if the system includes a slow CPU module the target-rotation-time should be set carefully. Since only 85 examples are of region high the weight of 38 is an indication for a high reliability also of this rule.

These results illustrate the applicability of the presented approach for metamodeling tasks. The approximation error indicates the reliability of the metamodel and the Fuzzy Graph can be used for new simulation experiments. The example rules deliver helpful information about dependencies between factors and the output of interest.

\section{Conclusions}

In this paper a new approach to handle complex simulation models through an analysis of data from experiments has been presented. The analysis is based on Fuzzy Graphs which makes it possible to use not only real valued but also soft or noisy values. This is especially well suited for the analysis of simulation data due to their stochastic character. It was demonstrated that rules from the Fuzzy Graph deliver meaningful information about the relation between input parameters and the output, a very helpful information when analyzing complex models like the used token bus. Since the presented method is easy to use and no parameters are needed, the proposed methodology allows to handle complex simulation models. 


\section{Acknowledgments}

We thank Prof. D. Schmid for his support and the opportunity to work on this interesting project. Thanks also to Markus Weihrauch, who helped with the actual implementation, and to Raffaele Carluccio, who built the queuing network of the token bus.

\section{References}

1. Michael R. Berthold and Klaus-Peter Huber. Building fuzzy graphs from examples. In IEEE International Conference on Fuzzy Systems, 1, pages 608-613, September 1996.

2. Linda W. Friedman and Israel Pressman. The metamodel in simulation analysis: Can it be trusted? Journal of the Operational Research Society, 39(10):939-948, 1988.

3. Charles M. Higgins and Rodney M. Goodman. Learning fuzzy rule-based neural networks for control. In Advances in Neural Information Processing Systems, 5, pages 350-357, California, 1993. Morgan Kaufmann.

4. Klaus-Peter Huber and Michael R. Berthold. Building precise classifiers with automatic rule extraction. In IEEE International Conference on Neural Networks, 3, pages 1263-1268, 1995.

5. Klaus-Peter Huber and Helena Szczerbicka. Sensitivity analysis of simulation models with decision tree algorithms. In Proceedings of the European Simulation Symposium ESS'94, volume 1, pages 43-47, 1994.

6. J.P.C. Kleijnen. Regression metamodels for generalizing simulation results. IEEE Transactions on Systems, Man and Cybernetics, 9(2):93-96, 1979.

7. Henri Pierreval. Rule-based simulation metamodels. European Journal of Operational Research, 61:6-17, 1992.

8. Patrick K. Simpson. Fuzzy min-max neural networks - part 2: Clustering. IEEE Transactions on Fuzzy Systems, 1(1):32-45, january 1993.

9. Volkmar Uebele, Shigeo Abe, and Ming-Shong Lan. A neural-network-based fuzzy classifier. IEEE Transactions on Systems, Man, and Cybernetics, 25(2), february 1995.

10. Li-Xin Wang and Jerry M. Mendel. Generating rules by learning from examples. In International Symposium on Intelligent Control, pages 263-268. IEEE, 1991.

11. Lotfi A. Zadeh. Soft computing and fuzzy logic. IEEE Software, pages 48-56, november 1994. 